\title{
Unemployment and the Durational Structure of Exit Rates
}

\author{
Karl Whelan \\ Division of Research and Statistics \\ Federal Reserve Board*
}

October 1997

\begin{abstract}
This paper presents a simple model of wage bargaining and employment flows designed to address the effects of policies to increase the rate of exit to employment of the long-term unemployed. Exit rates from long and short-term unemployment have two effects on the unemployment rate: a positive one as high exit rates strengthen current employees' bargaining positions and thus wages and a negative one as faster outflows from unemployment reduce the stock of unemployed. Thus, there is a trade-off between the exit rate from long-term unemployment and the exit rate from short-term unemployment. The paper's principal result is that, in steady-state, increasing the exit rate from long-term unemployment reduces the unemployment rate. Dynamic simulations show that raising the exit rate of the long-term unemployed leads to a decrease in both the mean and variance of the unemployment rate.
\end{abstract}

${ }^{*}$ This paper is a revised version of Chapter 2 of my Ph.D. dissertation at MIT. I am very grateful to Daron Acemoglu and Olivier Blanchard for comments. The views expressed in this paper are those of the author and do not necessarily reflect the views of the Board of Governors or the staff of the Federal Reserve System. Email: kwhelan@frb.gov. 


\section{Contents}

1 Introduction $\quad 3$

2 The Model $\quad 6$

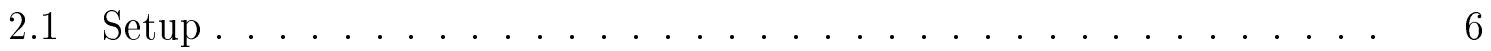

2.2 Exit Rates and the Outside Option ................ 8

2.3 Exit Rates and Unemployment Dynamics . . . . . . . . . . . . . 12

2.4 The Model's Equilibrium . . . . . . . . . . . . . 13

3 Properties of Steady-State Equilibria $\quad 14$

3.1 Unemployment and Exit Rates ................. 14

3.2 Quantitative Importance . . . . . . . . . . . . . 17

4 Extensions $\quad 19$

4.1 Labor Heterogeneity ....................... 19

4.2 Out-of-Steady-State Dynamics . . . . . . . . . . . . . . 21

5 Conclusion $\quad 23$

A Omitted Proof $\quad 25$ 


\section{Introduction}

The persistent high rate of long-term unemployment is perhaps the European Union's most serious economic problem and it is made all the more so by the well-known pattern of declining exit rates from unemployment as duration rises: those unemployed longest are the least likely to obtain employment. It is hardly surprising, then, that many European economists and policy-makers have been proposing policies aimed at increasing the exit rate to employment among the long-term unemployed. Examples of such policies include job training and placement programs for the long-term unemployed, as recommended by Layard (1997), and targeted employment subsidies, as proposed by Snower (1997).

The most obvious criticism of these policy recommendations is that they can merely obtain jobs for the long-term unemployed at the expense of the short-term unemployed, and so increase the rate of outflow from long-term unemployment at the expense of increasing the inflow. Proponents, however, have usually countered such criticisms with two arguments. Firstly, it is argued that increasing the employability of the longterm unemployed weakens the bargaining power of current employees and thus reduce wages and increases the number of jobs. ${ }^{1}$ Secondly, as Layard (1997) has pointed out, if government policies can make the long-term unemployed more employable without making the short-term unemployed less so, then they can increase the overall rate of outflow from unemployment and so, holding the rate of inflow constant, such policies can reduce the stock of unemployed.

This paper presents a simple but general model for analyzing the effects of policies which increase the exit rate to employment of the long-term unemployed. It focuses on the dual role which exit rates play in determining the unemployment rate: directly through their effect on employment flows and indirectly through their effect on wages.

\footnotetext{
${ }^{1}$ See Martin (1994) or Snower (1994) for versions of this argument.
} 
Wages are determined through a bargaining mechanism between the employer and the employee in which the employer needs to pay a wage which depends upon the employee's "outside option", usually represented as the expected present discounted value of earnings while short-term unemployed. The most obvious example of such a bargaining mechanism is the Shapiro-Stiglitz model of efficiency wages, but modelling wages as a function of employees' outside option has been a feature of almost all modern models of wage determination, including the Nash bargaining model of Layard and Nickell (1986). This approach, however, provides a simple counterpoint to the view that increasing the exit rate from long-term unemployment must create jobs by directly reducing wages. This is because wages are an increasing function of both short-term and long-term exit rates: raising either strengthens bargaining positions by increasing workers' outside option. Thus, holding all else equal, an increase in the exit rate from long-term unemployment raises wages and thus unemployment.

This paper's principal result, however, is that in steady-state equilibrium, policies which increase the exit rate from long-term unemployment do reduce the unemployment rate. Why? Because once we raise the exit rate from long-term unemployment, we cannot hold "all else equal": with an unchanged exit rate from short-term unemployment, these policies would also act to reduce the stock of unemployed by increasing the overall rate of outflow. Since this is incompatible with an increase in wages, labor market interventions which succeed in increasing long-term exit rates must do so at expense of reducing short-term exit rates and we show that they do so in a way that reduces wages and the unemployment rate.

The previous papers which are most closely related to this work are Shapiro and Stiglitz's (1984) model of efficiency wages and Blanchard and Diamond's (1994) discussion of the effect on wages of employer hiring rules which rank applicants by unemployment duration. The model in this paper shares the Shapiro-Stiglitz model's 
focus on the dual role of exit rates, affecting the unemployment rate indirectly through wages and directly through flow effects. However, while we calibrate an efficiency wage version of the model for illustrative purposes, this paper's results do not depend on other specifics of the efficiency wage model. Blanchard and Diamond compared wages determined by a Nash bargaining rule for two cases, one in which firms fill vacancies by choosing applicants randomly and one in which firms hire the applicant with the shortest unemployment duration. Their results showed that, for the same steady-state unemployment rate (which was independent of the wage level), wages were higher under durational ranking than under random hiring. Blanchard and Diamond's model featured an exogenous unemployment rate and allowed for comparison of wages under two specific firm hiring rules and under specific assumptions concerning matching between vacancies and the unemployed. The model in this paper, however, has an endogenously-determined unemployment rate and its results are very general. ${ }^{2}$

The contents of the paper are as follows. Section 2 outlines the basic structure of the model and Section 3 presents the principal results. Section 4 extends the model to account for labor heterogeneity and also looks at out-of-steady-state simulations. These show that increasing the exit rate of the long-term unemployed leads to lower peak levels of unemployment during recessions, faster recoveries and a lower average unemployment rate over the business cycle. Section 5 concludes.

\footnotetext{
${ }^{2}$ This generality comes at a price: the model in this paper features discrete time and two exit rates, one for short-term unemployed and one for long-term while Blanchard and Diamond's model is more realistic in featuring continous time and a contiuum of exit rates.
} 


\section{The Model}

\subsection{Setup}

Time is discrete. The economy has a fixed labor force normalized to 1.

Firms: There is a continuum of firms whose mass is also normalized to 1. All firms produce a homogenous good with price equal to one according to identical technologies, with each firm having a concave production function. There is no physical capital and we will start the analysis with the assumption that labor is homogenous. Thus, the production function is of the form $A F(L)$ where $A$ is a productivity parameter. In Section 4, we allow for the more realistic assumption of heterogenous labor inputs. Labor demand is determined by $F^{\prime}(L)=w$ where $w$ is the wage level.

Wages: Workers are risk-neutral and wish to maximize the expected present discounted value of income using discount rate $(1+r)^{-1}$. Wages are determined in a bargaining process in which the wage level depends positively on workers' "outside option", the expected present discounted value of being short-term unemployed, $V_{S}$, as well as on other variables influencing wage bargaining which we will label $z: w=g\left(V_{S}, z\right)$. For illustrative purposes, we will use a simple extension of the Shapiro-Stiglitz efficiency wage model as a specific example of such a bargaining process.

Separations: There is an exogenous separation process in which a fraction $b$ of each firm's workers become unemployed each period. ${ }^{3}$

Exit Rates: At the end of their first period of unemployment, workers at time $t$ obtain employment for the next period with probability $a_{S_{t}}$. If they do not obtain employment at the end of their first period, they become long-term unemployed. Once long-term unemployed they have exit rate $a_{L_{t+k}}$ at time $t+k$. These exit rates determine two

\footnotetext{
${ }^{3}$ Equivalently, we could assume that a fraction $b$ of firms fail each period.
} 
values for the unemployment rate, one through their effect on wages and thus labor demand and one through their direct effect on the stock of unemployed. Thus, the set of feasible values for these exit rates will be restricted by the necessity for these two generated unemployment rates to be equal. However, this one restriction on the two-dimensional set of possible exit rates still leaves a one-dimensional continuum of feasible exit rate combinations: which combination is observed can be influenced by government policies to reduce duration dependence.

Note that our assumption concerning exit rates is a very general one: it is merely a statement that there are separate exit rates for long and short-term unemployed. If skill loss during unemployment, declining search intensity with duration, or employer hiring rules which rank applicants by duration are important, then these exit rates will decline with duration. It is important to note, however, that there is an extensive empirical literature on the question of whether the duration dependence observed in aggregate exit rates is due to "genuine" duration dependence (as an individual's unemployment spell lengthens, her probability of exit from unemployment decreases) or labor heterogeneity (individuals with different characteristics have different exit rates and so the long-term unemployed consist disproportionately of those with low exit rates). That heterogeneity is an important influence on average exit rates is beyond doubt. It is well known that long-term unemployment rates differ by education and other observable characteristics. However, despite the large empirical literature on this topic, there appears to be little agreement on the relative importance of heterogeneity and genuine duration dependence. ${ }^{4}$ Thus, we do not make any assumption concerning whether exit rates display "genuine" duration dependence. However, our results below do imply that the size (although not the sign) of the effect on the unemployment rate of increasing

\footnotetext{
${ }^{4}$ For papers based on macro data, see Jackman and Layard (1991) for an argument in favor of the importance of genuine duration dependence and van den Berg and van Ours (1994) for a more mixed assessment. Devine and Kiefer (1991) review the similarly mixed results from micro data studies.
} 
exit rates from long-term unemployment depends upon how much duration dependence there was to start with: in other words, duration dependence affects the unemployment rate in a nonlinear fashion.

\subsection{Exit Rates and the Outside Option}

Consider now the determination of the value of employees' outside option, the value of being short-term unemployed, $V_{S}$. Define $V_{E}$ as the expected present discounted value of income obtained while employed. Without loss if generality, we will assume

that earnings while unemployed equal zero. The expected present discounted values of earnings while in short and long-term unemployment at time $t$ are given by

$$
\begin{aligned}
& V_{S_{t}}=\frac{1}{1+r}\left(a_{S_{t}} V_{E_{t+1}}+\left(1-a_{S_{t}}\right) V_{L_{t+1}}\right) \\
& V_{L_{t}}=\frac{1}{1+r}\left(a_{L_{t}} V_{E_{t+1}}+\left(1-a_{L_{t}}\right) V_{L_{t+1}}\right)
\end{aligned}
$$

Repeated substitution of these formulas, together with a bargaining equation linking wages and thus $V_{E}$ to $V_{S}$, yields a formula linking $V_{S_{t}}$ to positively to current and future exit rates:

$$
V_{S_{t}}=V\left(a_{S_{t}}, a_{L_{t+1}}, a_{S_{t+2}}, a_{L_{t+2}}, a_{S_{t+3}}, a_{L_{t+3}, \ldots \ldots}\right)
$$

Since firms' demand for labor depends negatively upon the wage level and positively upon the productivity parameter $A$ we have a general expression for the unemployment rate:

$$
U=U_{w}\left(a_{S_{t}}, a_{L_{t+1}}, a_{S_{t+2},}, a_{L_{t+2}}, a_{S_{t+3}}, a_{L_{t+3}, \ldots \ldots .,}, A\right)
$$

The unemployment rate depends positively upon all current and future exit rates, negatively upon $A$ and also upon the bargaining variable $z$.

This general formula for the unemployment rate will be very complicated. In steadystate, however, we have a simpler expression 


$$
U=H_{w}\left(a_{S}, a_{L}, z, A\right)
$$

whose crucial properties can be explained using the following result.

Result 1: In steady-state, the relative size of the effects of $a_{S}$ and $a_{L}$ on $V_{S}$, and thus $H_{w}$, depends upon the variable

$$
\theta_{w}=\frac{1-a_{S}}{r+a_{L}}
$$

since

$$
\frac{\partial V_{S}}{\partial a_{L}}=\theta_{w} \frac{\partial V_{S}}{\partial a_{S}}
$$

Proof : Dropping the time subscripts from equations 1 and 2 we get

$$
\begin{aligned}
V_{S} & =\frac{1}{1+r}\left(a_{S} V_{E}+\left(1-a_{S}\right) V_{L}\right) \\
V_{L} & =\frac{1}{1+r}\left(a_{L} V_{E}+\left(1-a_{L}\right) V_{L}\right)
\end{aligned}
$$

Solving for $V_{L}$ we obtain

$$
V_{L}=\frac{a_{L}}{r+a_{L}} V_{E}
$$

Inserting this into equation 3 gives us

$$
V_{S}=\frac{1}{1+r}\left(a_{S} V_{E}+\left(1-a_{S}\right)\left(\frac{a_{L}}{r+a_{L}} V_{E}\right)\right)
$$

So given the definition of $\theta_{w}$ we have

$$
V_{S}=\frac{a_{S}+a_{L} \theta_{w}}{1+r} V_{E}
$$

Differentiating this gives

$$
\begin{gathered}
\frac{\partial V_{S}}{\partial a_{S}}=\frac{V_{E}}{1+r}\left(1-\frac{a_{L}}{r+a_{L}}\right)=\frac{1}{r+a_{L}} \frac{r}{1+r} V_{E}>0 \\
\frac{\partial V_{S}}{\partial a_{L}}=\frac{V_{E}}{1+r}\left(\theta_{w}-\frac{a_{L}\left(1-a_{S}\right)}{\left(r+a_{L}\right)^{2}}\right)=\theta_{w} \frac{\partial V_{S}}{\partial a_{S}}
\end{gathered}
$$

as required. 
This result makes the simple point that those currently employed will not always prefer policies which act to increase $a_{S}$ to those which act to increase $a_{L}$. Which exit rate current employees prefer to see raised will depend upon the variable $\theta_{w}$ which in turn depends negatively on both short and long-term exit rates: the numerator term $\left(1-a_{S}\right)$ represents the fact that the higher $a_{S}$ is the less important $a_{L}$ will become while the denominator term $\left(r+a_{L}\right)^{-1}$ describes the effect of an increase in $a_{L}$ once a worker has failed to exit short-term unemployment. When $\theta_{w}>1$ long-term unemployment becomes a likely enough state for short-term unemployed individuals to find themselves in, perhaps for a number of periods, and so they care more about increasing the value of this state than they do about becoming re-employed next period.

\section{Example: Efficiency Wages}

One example of wage determination which is consistent with our assumptions is the Shapiro-Stiglitz model of efficiency wages, extended to include separate exit rates for short and long-term unemployed. Workers obtain flow utility $w$ when shirking and $w-e$ when not shirking. Each period there is a probability $b$ that a worker will lose her job and, independently, imperfect monitoring of workers implies an additional independent probability $q$ that workers who are shirking are caught and fired. The asset equations characterizing the expected present discounted value of utility obtained while shirking and not shirking are, respectively:

$$
\begin{gathered}
V_{E_{t}}^{s h}=w_{t}+\frac{1}{1+r}\left((b+q) V_{S_{t+1}}+(1-b-q) V_{E_{t+1}}^{s h}\right) \\
V_{E_{t}}=w_{t}-e+\frac{1}{1+r}\left(b V_{S_{t+1}}+(1-b) V_{E_{t+1}}\right)
\end{gathered}
$$

where $V_{S}$ is the value of being in short-term unemployment. Wages are always set so that $V_{E}^{s h}=V_{E}$ :

$$
w_{t}=\frac{r}{1+r} V_{S_{t+1}}+\left(\frac{r+b+q}{q}\right) e
$$


Assuming workers do not shirk the steady-state value of being employed is.

$$
V_{E}=\frac{1+r}{r+b}(w-e)+\frac{b}{r+b} V_{S}
$$

Inserting equation 6 into equation 7 and re-arranging we get

$$
V_{E}=V_{S}+\frac{(1+r) e}{q}
$$

So, together the parameters $r, e$ and $q$ determine the extent of "insider power" which imperfect monitoring gives workers.

Inserting equation 8 into equation 5 gives

$$
V_{S}=\frac{a_{S}+a_{L} \theta_{w}}{1+r}\left(V_{S}+\frac{(1+r) e}{q}\right)
$$

Re-arranging this gives,

$$
\frac{r}{1+r} V_{S}=\frac{1-r \theta_{w}}{1+\theta_{w}} \frac{e}{q}
$$

Thus, the efficiency wage can be written in terms of the model's parameters as

$$
w=\left(1+\frac{b}{q}+\frac{1+r}{q\left(1+\theta_{w}\right)}\right) e
$$

Note that now the parameter $\theta_{w}$ not only represents the relative effects of $a_{S}$ and $a_{L}$ on wages but also becomes a sufficient statistic for their effect. ${ }^{5}$ When $a_{S}=a_{L}=a$ this formula reduces to the familiar Shapiro-Stiglitz wage formula

$$
w=\left(1+\frac{a+b+r}{q}\right) e
$$

\footnotetext{
${ }^{5}$ This is because $\frac{\partial \theta_{w}}{\partial a_{L}}=\theta_{w} \frac{\partial \theta_{w}}{\partial a_{S}}$.
} 


\subsection{Exit Rates and Unemployment Dynamics}

We have described how future exit rates can affect the unemployment rate by influencing the bargaining position of workers and through this wages. Thus at time $t$ a high value of $a_{S_{t}}$ tends to produce high unemployment. However, once we reach time $t+1$ this high value of $a_{S_{t}}$ will tend to produce low unemployment because it has contributed directly to reducing the stock of unemployed individuals. More precisely, changes in stock of unemployed will be given by

$$
\Delta U_{t}=b L_{t-1}-a_{S_{t-1}} U_{S_{t-1}}-a_{L_{t-1}} U_{L_{t-1}}
$$

where $L$ is employment, $U_{S}$ is the number of short-term unemployed and $U_{L}$ is the number of long-term unemployed. Given the assumption that $U_{S_{t}}=b L_{t-1}$ we get a second-order difference equation in $U_{t}$

$$
U_{t}=U_{t-1}+b\left(1-U_{t-1}\right)-b a_{S_{t-1}}\left(1-U_{t-2}\right)-a_{L_{t-1}}\left(U_{t-1}-b\left(1-U_{t-2}\right)\right)
$$

Thus the unemployment rate at time $t$ is a function of all past exit rates and initial unemployment

$$
U=U_{f}\left(a_{S_{t-1}}, a_{L_{t-1}}, a_{S_{t-2}}, a_{L_{t-2}}, a_{S_{t-3}}, a_{L_{t-3}}, \ldots, b, U_{0}, U_{-1}\right)
$$

The steady-state solution to equation 10 is given by

$$
U=\frac{b\left(1+a_{L}-a_{S}\right)}{a_{L}+b\left(1+a_{L}-a_{S}\right)}
$$

or, defining a new variable

$$
\begin{gathered}
\theta_{f}=\frac{1-a_{S}}{a_{L}} \\
U=H_{f}\left(a_{S}, a_{L}, b\right)=\frac{b\left(1+\theta_{f}\right)}{1+b\left(1+\theta_{f}\right)}
\end{gathered}
$$


Note that since, in steady-state, $\left(1-a_{S}\right) U_{S}=a_{L} U_{L}$, the proportion of the unemployed who are in long-term unemployment is given by $1+\theta_{f}$. Equation 12 describes a monotonic relationship between $U$ and $1+\theta_{f}$. Thus, unless policies to increase the rate of exit from long-term unemployment can reduce the total stock of unemployed, they cannot affect the proportion of that stock who are long-term unemployed.

Along this steady-state unemployment schedule the effects of exit rates are:

$$
\begin{gathered}
\frac{\partial H_{f}}{\partial a_{S}}=\frac{\partial H_{f}}{\partial \theta_{f}} \frac{\partial \theta_{f}}{\partial a_{S}}=\frac{\partial H_{f}}{\partial \theta_{f}} \frac{-1}{a_{L}}<0 \\
\frac{\partial H_{f}}{\partial a_{L}}=\frac{\partial H_{f}}{\partial \theta_{f}} \frac{\partial \theta_{f}}{\partial a_{L}}=\frac{\partial H_{f}}{\partial \theta_{f}} \frac{-\left(1-a_{S}\right)}{a_{L}^{2}}<0
\end{gathered}
$$

These results make the intuitive point that for a given rate of inflow into unemployment, the higher are the rates of exit from unemployment then the lower the stock of unemployed will be. They also give us a counterpart to Result 1 .

Result 2: In steady-state, the relative size of the effects of exit rates on $H_{f}$ are determined by $\theta_{f}$. In other words,

$$
\frac{\partial H_{f}}{\partial a_{L}}=\theta_{f} \frac{\partial H_{f}}{\partial a_{S}}
$$

Thus, $a_{S}$ has a weaker effect in reducing unemployment than $a_{L}$ if and only if $\theta_{f}>1$, that is, when there are more long-term unemployed than short-term.

\subsection{The Model's Equilibrium}

The economy's equilibrium is characterized by a path for exit rates which yields

$U_{w}\left(a_{S_{t}}, a_{L_{t+1}}, a_{S_{t+2}}, a_{L_{t+2}}, a_{S_{t+3}}, a_{L_{t+3}, \ldots . .,}, A, z\right)=U_{f}\left(a_{S_{t-1}}, a_{L_{t-1}}, a_{S_{t-2}}, a_{L_{t-2}}, \ldots . a_{S_{0}}, a_{L_{0}}, U_{0}, U_{-1}\right)$ 
Thus, for $t=1,2, \ldots N$ we have $N$ simultaneous equations in $2 N$ exit rate variables. Clearly, this will have an infinite space of solutions. We pin down a unique equilibrium path for exit rates and unemployment by specifying a rule $a_{S_{t}}=h_{t}\left(a_{L_{t}}\right)$ which determines the pattern of duration dependence.

Characterizing the set of feasible steady-state equilibria consists of finding the set of $\left(a_{S}, a_{L}\right)$ combinations which give us

$$
U=H_{w}\left(a_{S}, a_{L}, A, z\right)=H_{f}\left(a_{S}, a_{L}, b\right)
$$

There is a continuum of possible solutions $\left(a_{S}, a_{L}\right)$ to this equation and an equation describing the relative structure of the exit rates is required to pin down a unique equilibrium. We can now investigate the properties of the set of feasible solutions to this equation.

\section{Properties of Steady-State Equilibria}

Equation 13 describes the determination of steady-state equilibrium unemployment. Are there are any important differences between the different possible solutions to the equation. In particular, does a reduction in the extent of duration dependence reduce the equilibrium unemployment rate? Our main result shows that it does.

\subsection{Unemployment and Exit Rates}

Firstly, it is clear that there is a trade-off between $a_{S}$ and $a_{L}$. 
Result 3: Let $A, b$ and $z$ be constant. Then, in the set of steady-state equilibrium solutions there exists an implicit functional relationship $a_{S}=g\left(a_{L}\right)$ where $g^{\prime}\left(a_{L}\right)<0$.

Proof: From equation 13 we have an equality between $H_{w}$ and $H_{f}$. Since we are taking all non-exit rate parameters to be constant suppress these arguments to obtain

$$
H_{w}\left(a_{S}, a_{L}\right)-H_{f}\left(a_{S}, a_{L}\right)=0
$$

This gives a constraint which we can differentiate with respect to $a_{L}$ to obtain an implicit function $a_{S}=g\left(a_{L}\right)$ :

$$
\begin{gathered}
\frac{\partial H_{w}}{\partial a_{L}}+\frac{\partial H_{w}}{\partial a_{S}} \frac{d a_{S}}{d a_{L}}-\frac{\partial H_{f}}{\partial a_{L}}-\frac{\partial H_{f}}{\partial a_{S}} \frac{d a_{S}}{d a_{L}}=0 \\
\Rightarrow g^{\prime}\left(a_{L}\right)=\frac{d a_{S}}{d a_{L}}=-\frac{\frac{\partial H_{w}}{\partial a_{L}}-\frac{\partial H_{f}}{\partial a_{L}}}{\frac{\partial H_{w}}{\partial a_{S}}-\frac{\partial H_{f}}{\partial a_{S}}}<0
\end{gathered}
$$

as required.

This proposition can by illustrated by simulating the two exit rate efficiency wage model derived above. The following choice of parameter values were used: $A=1, b=$ $0.0566, r=0.125, e=0.2, q=0.25, F(L)=L^{0.7}$. Since $\frac{b}{1+b}$ represents the infinum of the set of feasible unemployment rates, $b=0.04166$ was chosen to give this a value of .04. The values of $r, e$ and $q$ together imply a value of 0.75 for $V_{E}-V_{S}$. This is consistent with $V_{E}$ being between $20 \%$ and $21 \%$ higher than $V_{S}$. Thus, the simulations describe an economy which cannot have unemployment lower than $4 \%$ and in which workers have significant insider power.

Figure 1 shows the set of feasible combinations of $a_{S}$ and $a_{L}$. The graph shows a strong, almost exactly linear, pattern of declining $a_{S}$ as $a_{L}$ gets larger. Repeated simulations show that this essentially linear pattern occurs for all sets of exogenous parameters. It can be easily shown that an increase in $e$ or $r$ will shift this schedule 
of feasible exit rates upwards, while an increase in $A$ or $q$ shifts it downwards. Thus, whatever rule is determining the relative structure of exit rates to close the model, an increase in $e$ or $r$ will result in higher unemployment and at least one exit rate being lower while an increase in $A$ or $q$ will have the opposite effect. ${ }^{6}$

We can now show that anything which increases the exit rate of the long-term unemployed will in fact decrease the equilibrium unemployment rate.

Result 4: Let $A, b$ and $z$ be constant. Then the steady-state equilibrium rate of unemployment $U$ depends negatively on $a_{L}$.

Proof: We have established the existence of a negative functional relationship between $a_{S}$ and $a_{L}$ such that $H_{w}\left(a_{S}\left(a_{L}\right), a_{L}\right)=H_{f}\left(a_{S}\left(a_{L}\right), a_{L}\right)=U$ and calculated its derivative. Given this we can calculate the sign of the change in unemployment when $a_{L}$ increases by differentiating either of the two functions $H_{w}$ or $H_{f}$. Take $H_{w}$.

$$
\frac{d U}{d a_{L}}=\frac{\partial H_{w}}{\partial a_{S}} \frac{d a_{S}}{d a_{L}}+\frac{\partial H_{w}}{\partial a_{L}}
$$

Inserting the formula in equation 14 we obtain

$$
\frac{d U}{d a_{L}}=\frac{\partial H_{w}}{\partial a_{S}}\left(\frac{\frac{\partial H_{f}}{\partial a_{L}}-\frac{\partial H_{w}}{\partial a_{L}}}{\frac{\partial H_{w}}{\partial a_{S}}-\frac{\partial H_{f}}{\partial a_{S}}}\right)+\frac{\partial H_{w}}{\partial a_{L}}
$$

Since we know that

$$
\frac{\partial H_{w}}{\partial a_{S}}-\frac{\partial H_{f}}{\partial a_{S}}>0
$$

we can multiply through by this to obtain the requirement that the derivative of $U$ with respect to $a_{L}$ is negative if and only if

\footnotetext{
${ }^{6}$ Unemployment depending positively on productivity is obviously a counter-factual implication since the unemployment rate is a bounded variable whereas productivity grows over time. This implication can be overturned, however, if we link unemployment benefits to productivity.
} 


$$
\begin{gathered}
\frac{\partial H_{w}}{\partial a_{S}}\left(\frac{\partial H_{f}}{\partial a_{L}}-\frac{\partial H_{w}}{\partial a_{L}}\right)+\frac{\partial H_{w}}{\partial a_{L}}\left(\frac{\partial H_{w}}{\partial a_{S}}-\frac{\partial H_{f}}{\partial a_{S}}\right)<0 \\
\Longrightarrow \frac{\partial H_{w}}{\partial a_{S}} \frac{\partial H_{f}}{\partial a_{L}}-\frac{\partial H_{w}}{\partial a_{L}} \frac{\partial H_{f}}{\partial a_{S}}<0
\end{gathered}
$$

Now use Results 1 and 2 to simplify this expression since

$$
\begin{aligned}
& \frac{\partial H_{w}}{\partial a_{L}}=\theta_{w} \frac{\partial H_{w}}{\partial a_{S}} \\
& \frac{\partial H_{f}}{\partial a_{L}}=\theta_{f} \frac{d H_{f}}{d a_{S}}
\end{aligned}
$$

Thus the required condition reduces to

$$
\frac{\partial H_{w}}{\partial a_{S}} \frac{\partial H_{f}}{\partial a_{S}}\left(\theta_{f}-\theta_{w}\right)<0 \Rightarrow \frac{\partial H_{w}}{\partial a_{S}} \frac{\partial H_{f}}{\partial a_{S}}\left(\frac{1-a_{S}}{a_{L}}-\frac{1-a_{S}}{r+a_{L}}\right)<0
$$

which is true.

So, increasing the long-term exit rate reduces the equilibrium unemployment rate as long as workers discount future earnings. The result holds because the relative size of the long-term exit rate's effect on the stock of unemployed through raising outflows is always larger then the relative size of its effect on wages. Even when an increase in $a_{L}$ has a stronger effect on wages than an increase in $a_{S}$, this can only be the case if there are more long-term unemployed than short-term unemployed and so the flow

effect outweighs the wage effect: an increase in $a_{L}$ always requires a sufficient fall in $a_{S}$ to reduce wages.

\subsection{Quantitative Importance}

We have shown that reducing duration dependence in exit rates can reduce the unemployment rate. However, when is the effect quantitatively important? Technically, 
what we are interested in is the partial derivatives of $\frac{d U}{d a_{L}}$ with respect to parameters of interest. Unfortunately these derivatives are very complicated and unsignable analytically. However, simulating the efficiency wage model, the size of the effect can be easily understood in terms of the term driving the result:

$$
\theta_{w}-\theta_{f}=d\left(a_{S}, a_{L}, r\right)
$$

If the function $d\left(a_{S}, a_{L}, r\right)$ equals zero then duration dependence has no effect on unemployment. Note then that the effect of $a_{S}$ on this function is negative and linear, the effect of $a_{L}$ is negative and convex and the effect of $r$ is positive and convex. ${ }^{7}$ This implies that the magnitude of duration dependence's effect on unemployment depends negatively on exit rates: the more exogenous parameters, such as those influencing insider power, are set so that the unemployment rate is high and exit rates are low, the stronger will be the effect of duration dependence. We can see this from Figure 2: this shows the relationship between unemployment and the difference between $a_{S}$ and $a_{L}$ for two different sets of parameter values: one with $e=0.25$, one with $e=0.22$, all other parameters as above. Both show a convex relationship between unemployment and duration dependence as the long-term exit rate falls. However, the relationship is more convex for the higher value of $e$ because exit rates are lower.

These results have two implications. Firstly, policies to reduce duration dependence will have their greatest impact on unemployment in economies such as those in Europe

\footnotetext{
${ }^{7}$ More specifically

$$
\begin{array}{cc}
\frac{\partial d}{\partial a_{S}}<0 & \frac{\partial^{2} d}{\partial a_{S}^{2}}=0 \\
\frac{\partial d}{\partial a_{S}}<0 & \frac{\partial^{2} d}{\partial a_{L}^{2}}=2\left(1-a_{S}\right)\left(\frac{1}{a_{L}^{3}}-\frac{1}{\left(r+a_{L}\right)^{3}}\right)>0 \\
\frac{\partial d}{\partial r}>0 & \frac{\partial^{2} d}{\partial r^{2}}=\frac{2\left(1-a_{S}\right)}{\left(r+a_{L}\right)^{3}}>0
\end{array}
$$
}


which currently have high unemployment. Secondly, the potential for a decline in the unemployment rate from policies to increase long-term exit rates will depend positively on the current extent of duration dependence. The simulations show however that the likely impact on unemployment of policies to increase the exit rate of the long-term unemployed is a small one. While bringing duration dependence down from very high rates reduces the unemployment rate by multiple points, Figure 2 shows the effect of moving the difference between long and short-term exit rates from the more realistic value of 0.3 to -0.3 is 0.6 for $e=0.25(9.4 \%$ to $8.8 \%)$ and 0.3 for $e=0.22$ (7.4\% to 7.1\%). Repeated simulations show that for all reasonable parameterizations of duration dependence and the unemployment rate, the effect is below one percentage point.

\section{Extensions}

Two questions can be raised about the results just derived. Firstly, given the obvious empirical importance of labor heterogeneity, can we draw much from an analysis based on homogenous labor? Secondly, are the insights gained from the steady-state results useful when thinking about an economy with business cycles? This section looks at these issues in turn.

\subsection{Labor Heterogeneity}

A standard way to introduce labor heterogeneity is to assume that differing types of labor enter into the production function in differing fashions. For simplicity, assume there are two distinct forms of labor input $F\left(L_{1}, L_{2}\right)$, and labor demand is determined by the standard first-order conditions $A \frac{\partial F}{\partial L_{i}}=w_{i}$. Labor turnover and wage determination are as before with a separate $V_{S}^{i}$ for each labor category. Depending on the quantity of each category of labor supplied and the marginal productivity of each category, there will 
be two classes of individuals with differing unemployment and exit rates. Assume that the exit rates from both categories display some level of genuine duration dependence. Letting $a_{S}^{1}$ and $a_{S}^{2}$ be the two short-term exit rates and $a_{L}^{1}$ and $a_{L}^{2}$ be the long-term exit rates, we thus have

$$
\begin{aligned}
& a_{S}^{1}=a_{L}^{1}+\sigma_{1} \geq 0 \\
& a_{S}^{2}=a_{L}^{2}+\sigma_{2} \geq 0
\end{aligned}
$$

where $\sigma_{1}$ and $\sigma_{2}$ are measures of duration dependence in the two labor markets. The following result explains the effects of these parameters on employment.

Result 5: Let $A, b$ and $z$ be constant. Then for a concave production function $F\left(L_{1}, L_{2}\right)$, in steady-state we have $\frac{d L_{1}}{d \sigma_{1}}, \frac{d L_{2}}{d \sigma_{2}}$ are negative while $\frac{d L_{1}}{d \sigma_{2}}, \frac{d L_{2}}{d \sigma_{1}}$ are negative if and only if $\frac{\partial^{2} F}{\partial L_{1} \partial L_{2}}>0$.

Proof: In appendix.

This result holds because segmented labor markets imply that the effects of type- $i$ exit rates on wages are exactly as before: an increase in $\sigma_{i}$ implies higher $w_{i}$ for constant $L_{i}$. Thus the effects of increased duration dependence are similar to the standard results for the effects on factor demand of an increase in a factor price and so unsurprising.

The implications of heterogenous labor can be seen from simulating this model. Specifically, the model was simulated using separate efficiency wages for each category of labor under the assumptions that the supply of each equals 0.5 and technology is CES

$$
F\left(L_{1}, L_{2}\right)=A\left(\delta_{1} L_{1}^{\rho}+\delta_{2} L_{2}^{\rho}\right)^{\frac{\alpha}{\rho}}
$$

where $\delta_{1}=0.75, \delta_{2}=1$ and $\rho=\frac{2}{3}$ : this number for $\rho$ comes from Hamermesh's (1993) estimate of $\sigma=3$ for the elasticity of substitution between skilled and unskilled 
labor. Thus category 1 is the unskilled (low wage, high unemployment) group. All other parameters are as before including $\alpha=0.7$. The cross partial derivative is

$$
\frac{\partial^{2} F}{\partial L_{1} \partial L_{2}}=A \delta_{1} \delta_{2} \alpha(\alpha-\rho)\left(L_{1} L_{2}\right)^{\rho-1}\left(\delta_{1} L_{1}^{\rho}+\delta_{2} L_{2}^{\rho}\right)^{\frac{\alpha}{\rho}-2}>0
$$

and so an increase in duration dependence in category $i$ will increase in unemployment in both categories with the effect on category $j$ being very small. However, because $\alpha-\rho$ is small the effects of $\sigma_{i}$ on $L_{j}$ turn out to be very small. Thus, the dominant effect on unemployment of an increase in $\sigma_{i}$ is the decrease in $L_{i}$. Figure 3 shows the unemployment rate for skilled and unskilled categories as we increase both $\sigma_{1}$ and $\sigma_{2}$.

This approach produces an interesting cross-sectional counterpart to our earlier result that policies to increase long-term exit rates have their strongest effects in depressed economies. Figure 3 shows that policies to increase the long-term exit rate have their strongest effect among the low-wage, high-unemployment category, with the explanation being analogous to the cross-country argument above.

\subsection{Out-of-Steady-State Dynamics}

The results derived thus far indicate that, for an economy in steady-state, policies to increase the exit rate of the long-term unemployed will lead to lower unemployment. While obviously suggestive, these results may have limited applicability to the real world since real economies cannot be characterized as displaying steady-state behavior: we are thus interested in how duration dependence affects an economy which goes through cyclical fluctuations.

Keeping the simple assumption that $a_{S}=a_{L}+\beta$ the time path for exit rates and thus unemployment is obtained by solving the $N$ simultaneous non-linear equations

$U_{f}\left(a_{S_{t-1}}, a_{S_{t-2}}, a_{S_{t-3}}, \ldots . ., a_{S_{0}}, U_{0}, U_{-1}\right)=U_{w}\left(a_{S_{t+1}}, a_{S_{t+2}}, a_{S_{t+3}}, \ldots \ldots ..\right) \quad t=1,2, \ldots . N$ 
for the $N$ variables $a_{S_{1}}, a_{S_{2}}, \ldots ., a_{S_{N}}$ supplying the initial conditions $a_{0}$ and $U_{0}$ as well as the terminal value for $V_{S_{N+1}}$ (and thus $U_{N}$ ). It is clear that there will be no analytical solution to these equations and so we need to apply numerical simulation methods. The model was solved using the Fair-Taylor algorithm for the solution of rational expectation models (Fair and Taylor, 1983).

Figures 4 and 5 describe two different types of out-of-steady-state behavior resulting from cyclical fluctuations in the productivity parameter $A$. With the parameter values used for the simulations and $A=1$ both economies are relatively depressed: the random hiring steady-state unemployment rate is $13.7 \%$ while the duration dependence rate is $14 \%$.

Stochastic Business Cycle: Figure 4 shows an extract from a simulation in which the exogenous productivity parameter $A$ goes through stochastic fluctuations. In particular, we set

$$
A=1+v_{t} \quad v_{t}=0.5 v_{t-1}+e_{t} \quad e_{t} \sim N(0,0.0075)
$$

Calculating moments over all time periods of the simulation, we find that the difference between mean unemployment rates is almost exactly equal to the difference between steady-state rates when evaluated at the mean of the $A$ process: this indicates that, despite the complexity of the model, the steady-state analysis still provides a useful baseline. The economy with duration dependence also has a higher variance for unemployment: 1.56 percentage points as opposed to 1.24. Figure 4 shows that the difference between the two economies is most pronounced during and after recessions with the duration dependence economy having higher peak values for unemployment. This economy has a higher variance for unemployment because during booms it has very similar levels of unemployment to the random hiring one. 
Deterministic Business Cycle: The model has also been simulated with $A$ displaying a deterministic cyclical pattern in which it equals 1 plus a sine wave with amplitude 0.02 and a cycle of 10 periods. Figure 5 shows the time-line unemployment using a 20 period sample. The results show that the economy with duration dependence moves more quickly to peak unemployment during recession and experiences a higher peak level of unemployment: in the depicted simulation, peak unemployment is 1 point higher. During the recovery period, the decline in unemployment is slower in the economy with duration dependence: the largest difference between the two unemployment rates, 1.5 points, occurs 4 periods after peak unemployment. Once the economy moves into its

boom period, however, the duration dependence economy experiences a lower level of unemployment at the bottom of the cycle: there is one period at the bottom of the cycle in which unemployment is a half point lower than the random hiring economy. Again the duration dependence economy has a higher average unemployment rate over the business cycle with the difference being close to the difference between steady-state rates evaluated at $A=1$.

\section{Conclusion}

This paper has shown how policies directed towards increasing the exit rate of the long-term unemployed can reduce total unemployment and that this result holds very generally. Sensitivity analysis of the results shows that the size of this effect depends positively on the rate of time-discounting, the current level of duration dependence and also on any structural variables which affects the unemployment rate positively. An implication of this result is that policies to increase long-term exit rates will be most effective in economies such as those in Europe which have structural factors underlying high unemployment and large numbers of long-term unemployed with low levels of labor market attachment. A cross-sectional implication is that policies directed at reducing 
duration dependence will be most effective in reducing unemployment when targeted at low-skill, high unemployment groups.

It must be noted, however, that simulations indicate that for realistic parameter values, the steady-state effect of these policies are small: certainly less than one percentage point. Finally, the out-of-steady-state simulations show that even for economies in which the steady-state gains from eliminating duration dependence are small, the effects on the cyclical behavior of unemployment can be substantial: the simulations suggested that economies with duration dependence have higher peak levels of unemployment, slower recoveries and higher average unemployment over the business cycle.

\section{References}

[1] Blanchard O.J. and P. Diamond (1994). "Ranking, Unemployment Duration and Wages", Review of Economic Studies, 61, 417-434.

[2] Devine T. and N. Kiefer (1991). Empirical Labor Economics, Oxford: Oxford University Press.

[3] Fair R. and J.B. Taylor (1983). "Solution and Maximum Likelihood Estimation of Dynamic Rational Expectation Models", Econometrica, 51, 1169-1185.

[4] Hamermesh D. (1993). Labor Demand, Princeton: Princeton University Press.

[5] Jackman R. and R. Layard (1991). "Does Long-Term Unemployment Reduce a Person's Chance of a Job", Economica, 93-106.

[6] Layard R. (1997). "Preventing Long-Term Unemployment: An Economic Analysis", in Unemployment Policy: Government Options for the Labor Market, Snower and de la Dehesa (eds.), Cambridge: Cambridge University Press. 
[7] Layard R., S. Nickell and R. Jackman (1991). Unemployment: Macroeconomic Performance and the Labour Market, Oxford: Oxford University Press.

[8] Layard R. and S. Nickell (1986). "Unemployment in Britain", Economica, 53:S121169.

[9] Martin J. (1994). "The Extent of High Unemployment in OECD Countries" in Reducing Unemployment: Current Issues and Policy Options, Federal Reserve Bank of Kansas City.

[10] Shapiro C. and J. Stiglitz (1984). "Equilibrium Unemployment as a WorkerDiscipline Device", American Economic Review, 433-444.

[11] Snower D. (1994). "Converting Unemployment Benefits into Employment Subsidies", American Economic Review, Papers and Proceedings, 84, 65-70.

[12] Snower D. (1997). "The Simple Economics of Benefit Transfers", in Unemployment Policy: Government Options for the Labor Market, Snower and de la Dehesa (eds.), Cambridge: Cambridge University Press.

[13] van den Berg G. and J. van Ours (1994). "Unemployment Dynamics and Duration Dependence in France, the Netherlands and the UK". Economic Journal, 104, 423-444.

\section{A Omitted Proof}

Proof of Result 5: For both types of labor we have

$$
N_{i}-L_{i}=\left(1+\frac{1-a_{L}^{i}-\sigma_{i}}{a_{L}^{i}}\right) b L_{i}
$$


This can be re-arranged to give

$$
a_{i}=\frac{b L_{i}\left(1-\sigma_{i}\right)}{N_{i}-L_{i}}
$$

Now the formula for the outside option for each type, $V_{S}^{i}\left(a_{S}^{i}, a_{L}^{i}\right)$ will be exactly as derived in Section 2.1. Thus, given a formula $w_{i}=g\left(V_{S}\right)$ where $g^{\prime}>0$, we can find the effect of $\sigma_{i}$ on $w_{i}$ as follows

$$
\begin{aligned}
\frac{\partial w_{i}}{\partial \sigma_{i}} & =g^{\prime}\left(V_{S}^{i}\right)\left(\frac{\partial V_{S}^{i}}{\partial a_{S}^{i}} \frac{\partial a_{S}^{i}}{\partial \sigma_{i}}+\frac{\partial V_{S}^{i}}{\partial a_{L}^{i}} \frac{\partial a_{L}^{i}}{\partial \sigma_{i}}\right) \\
& =g^{\prime}\left(V_{S}^{i}\right) \frac{\partial V_{S}^{i}}{\partial a_{S}^{2}}\left(\frac{\partial a_{S}^{i}}{\partial \sigma_{i}}+\theta_{w, i} \frac{\partial a_{L}^{i}}{\partial \sigma_{i}}\right) \\
& =g^{\prime}\left(V_{S}^{i}\right) \frac{\partial V_{S}^{i}}{\partial a_{S}^{i}}\left(1-\frac{b L_{i}}{N_{i}-L_{i}}+\frac{\theta_{w, i} b L_{i}}{N_{i}-L_{i}}\right)
\end{aligned}
$$

Using the steady-state conditions we have

$$
\frac{b L_{i}}{N_{i}-L_{i}}=\frac{1}{1+\frac{1-a_{S}^{i}}{a_{L}^{i}}}=\frac{1}{1+\theta_{f, i}}
$$

Thus

$$
\frac{\partial w_{i}}{\partial \sigma_{i}}=g^{\prime}\left(V_{S}^{i}\right) \frac{\partial V_{S}^{i}}{\partial a_{S}^{i}} \frac{1}{1+\theta_{f, i}}\left(\theta_{f, i}-\theta_{w, i}\right)>0
$$

Similarly we have $\frac{\partial w_{i}}{\partial L_{i}}>0$. Thus we can formulate the wage rate in category $i$ as $w_{i}=w_{i}\left(L_{i}, \sigma_{i}\right)$. Thus, the first-order conditions determining both labor inputs are

$$
\begin{aligned}
& \phi_{1}\left(L_{1}, L_{2}, \sigma_{1}\right)=A \frac{\partial F}{\partial L_{1}}-w_{1}\left(L_{1}, \sigma_{1}\right)=0 \\
& \phi_{2}\left(L_{1}, L_{2}, \sigma_{2}\right)=A \frac{\partial F}{\partial L_{2}}-w_{2}\left(L_{2}, \sigma_{2}\right)=0
\end{aligned}
$$

We are interested in the effects on $L_{1}$ and $L_{2}$ of changes in $\sigma_{1}$ and $\sigma_{2}$ and we have 2 nonlinear equations in these 4 variables. Use implicit differentiation to obtain

$$
\left(\begin{array}{ll}
\frac{\partial \phi_{1}}{\partial L_{1}} & \frac{\partial \phi_{1}}{\partial L_{2}} \\
\frac{\partial \phi_{2}}{\partial L_{1}} & \frac{\partial \phi_{2}}{\partial L_{2}}
\end{array}\right)\left(\begin{array}{c}
\frac{d L_{1}}{d \sigma_{i}} \\
\frac{d L_{2}}{d \sigma_{i}}
\end{array}\right)=-\left(\begin{array}{c}
\frac{\partial \phi_{1}}{\partial \sigma_{i}} \\
\frac{\partial \phi_{2}}{\partial \sigma_{i}}
\end{array}\right)
$$


and obtain solutions using Cramer's Rule. Letting

$$
D=\left(\begin{array}{ll}
\frac{\partial \phi_{1}}{\partial L_{1}} & \frac{\partial \phi_{1}}{\partial L_{2}} \\
\frac{\partial \phi_{2}}{\partial L_{1}} & \frac{\partial \phi_{2}}{\partial L_{2}}
\end{array}\right)
$$

we have solutions

$$
\begin{aligned}
& \frac{d L_{1}}{d \sigma_{i}}=\frac{-1}{|D|}\left|\begin{array}{cc}
\frac{\partial \phi_{1}}{\partial \sigma_{i}} & \frac{\partial \phi_{1}}{\partial L_{2}} \\
\frac{\partial \phi_{2}}{\partial \sigma_{i}} & \frac{\partial \phi_{2}}{\partial L_{2}}
\end{array}\right| \\
& \frac{d L_{2}}{d \sigma_{i}}=\frac{-1}{|D|}\left|\begin{array}{ll}
\frac{\partial \phi_{1}}{\partial L_{1}} & \frac{\partial \phi_{1}}{\partial \sigma_{i}} \\
\frac{\partial \phi_{2}}{\partial L_{1}} & \frac{\partial \phi_{2}}{\partial \sigma_{i}}
\end{array}\right|
\end{aligned}
$$

Let $H$ be the Hessian matrix of $F$. Then

$$
D=H-\left(\begin{array}{cc}
\frac{\partial w_{1}}{\partial L_{1}} & 0 \\
0 & \frac{\partial w_{2}}{\partial L_{2}}
\end{array}\right)
$$

The strict concavity of the production function gives us that $H$ is negative definite. Thus $D$ is the sum of two negative definite symmetric matrices and so is symmetric negative definite. This means that $|D|>0$.

Finally we get that

$$
\operatorname{Sign}\left(\frac{d L_{1}}{d \sigma_{1}}\right)=-\operatorname{Sign}\left(\frac{\partial \phi_{1}}{\partial \sigma_{1}} \frac{\partial \phi_{2}}{\partial L_{2}}\right)=-\operatorname{Sign}\left(\left(-\frac{\partial w}{\partial \sigma_{1}}\right)\left(\frac{\partial^{2} F}{\partial L_{2}^{2}}-\frac{\partial w_{2}}{\partial L_{2}}\right)\right)<0
$$

and so by symmetry $\frac{d L_{2}}{d \sigma_{2}}<0$. Similarly we have

$$
\operatorname{Sign}\left(\frac{d L_{1}}{d \sigma_{2}}\right)=\operatorname{Sign}\left(\frac{\partial \phi_{1}}{\partial L_{2}} \frac{\partial \phi_{2}}{\partial \sigma_{2}}\right)=\operatorname{Sign}\left(-\frac{\partial^{2} F}{\partial L_{1} \partial L_{2}} \frac{\partial w_{2}}{\partial \sigma_{2}}\right)<0
$$

by our assumption that $\frac{\partial^{2} F}{\partial L_{1} \partial L_{2}}>0$, and so also by symmetry we have $\frac{d L_{2}}{d \sigma_{1}}<0$. 


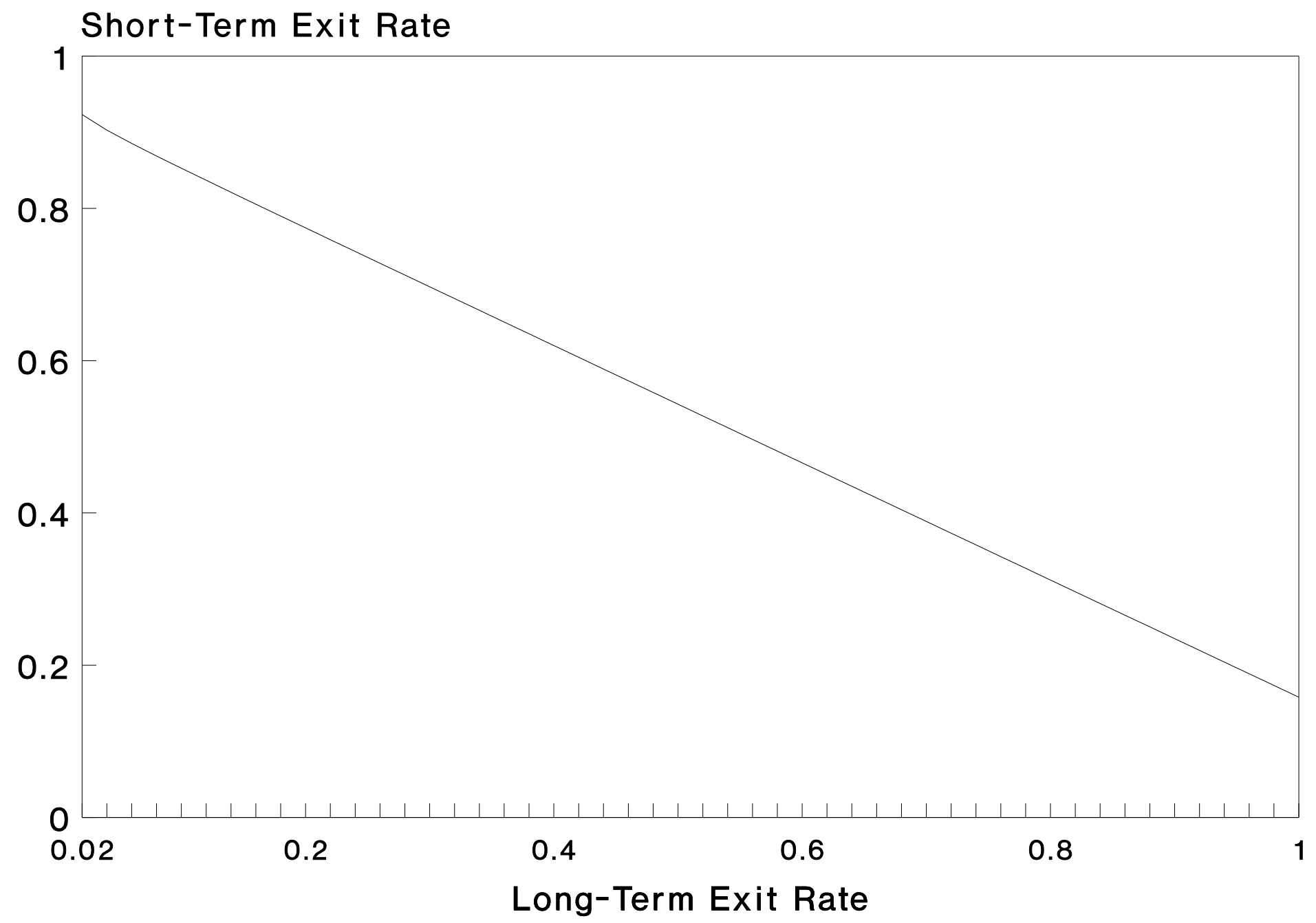

Figure 1: The Tradeoff Between Exit Rates 


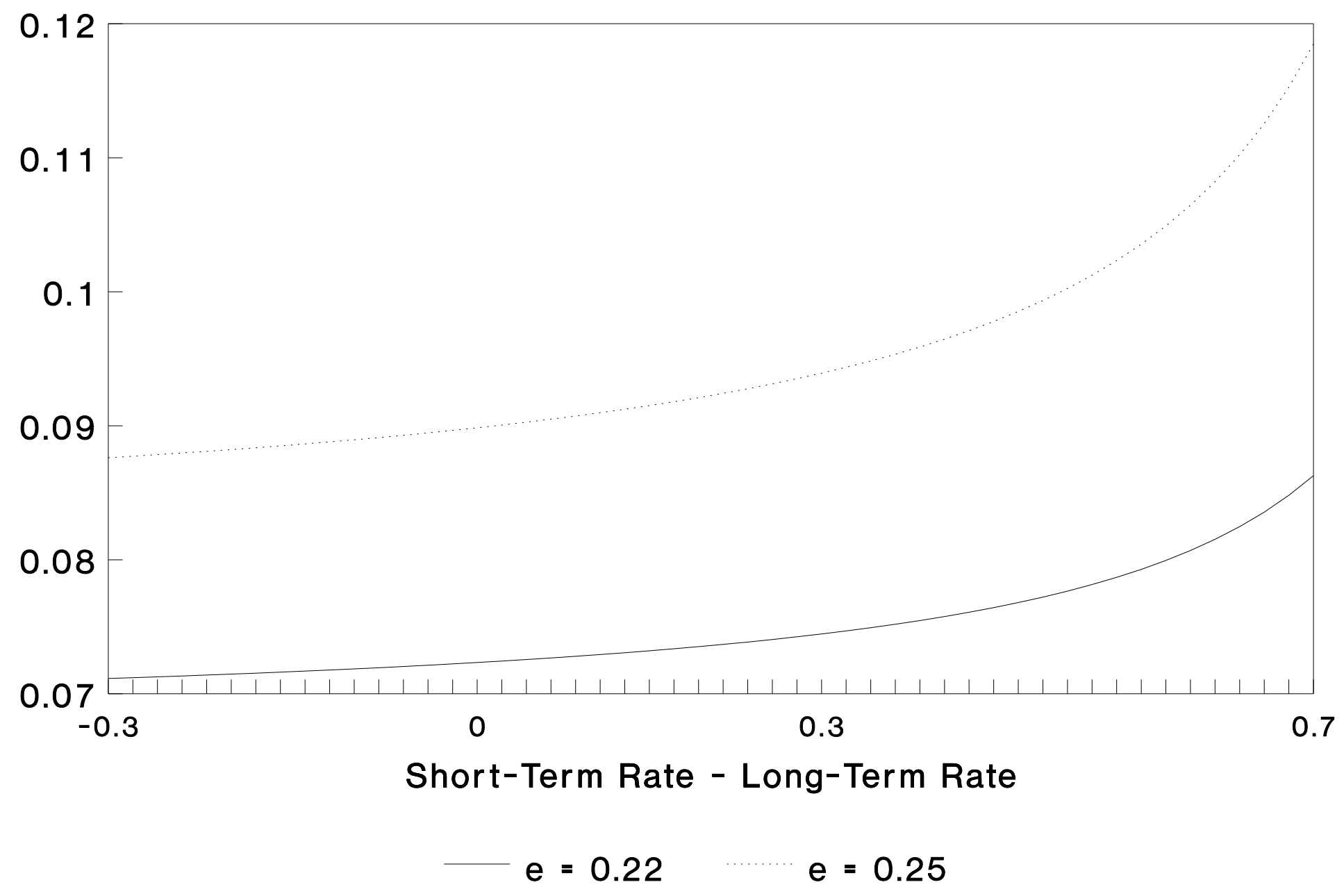

Figure 2: Steady-State Unemployment Rates and Duration Dependence 


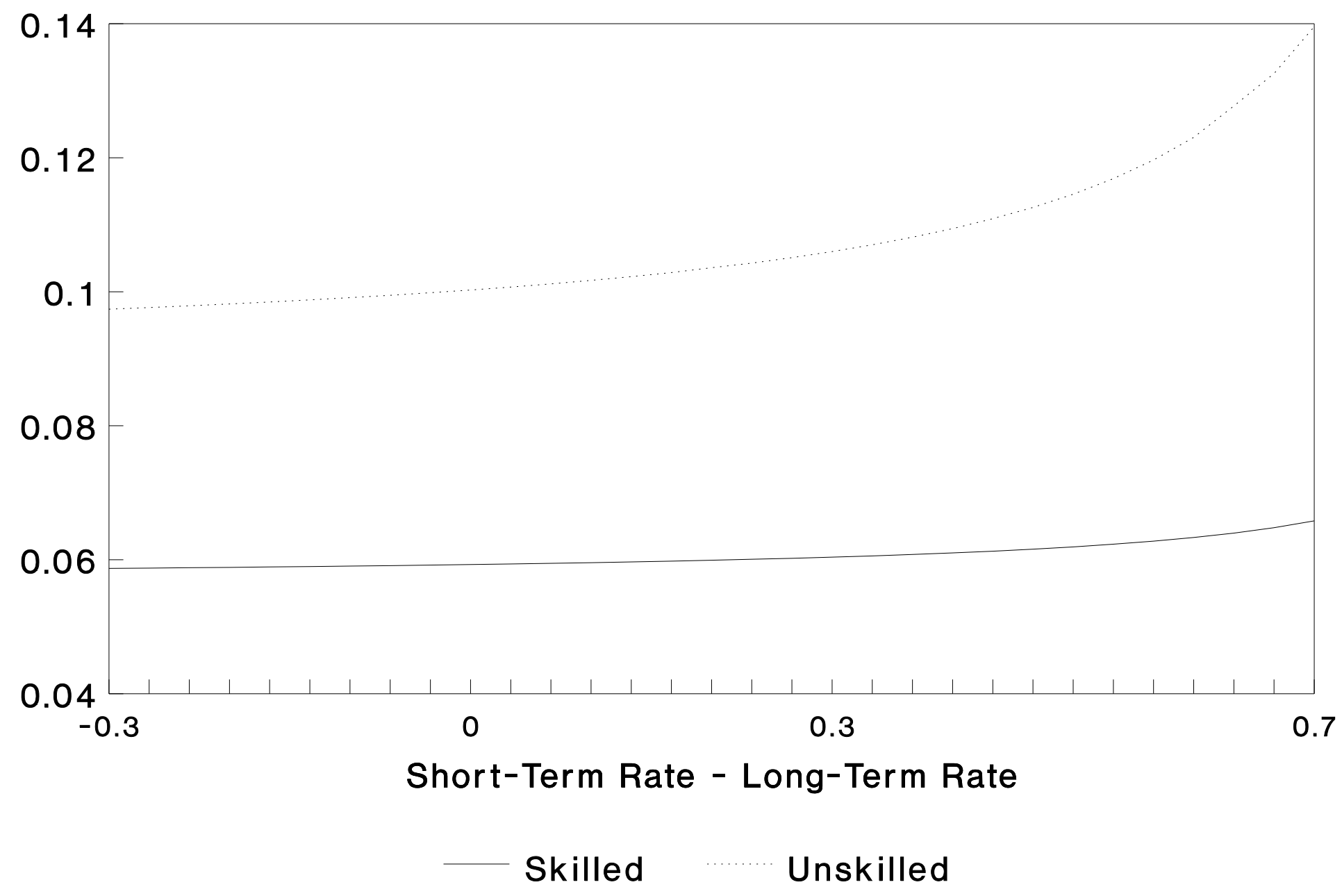

Figure 3: Skilled and Unskilled Steady-State Unemployment Rates 


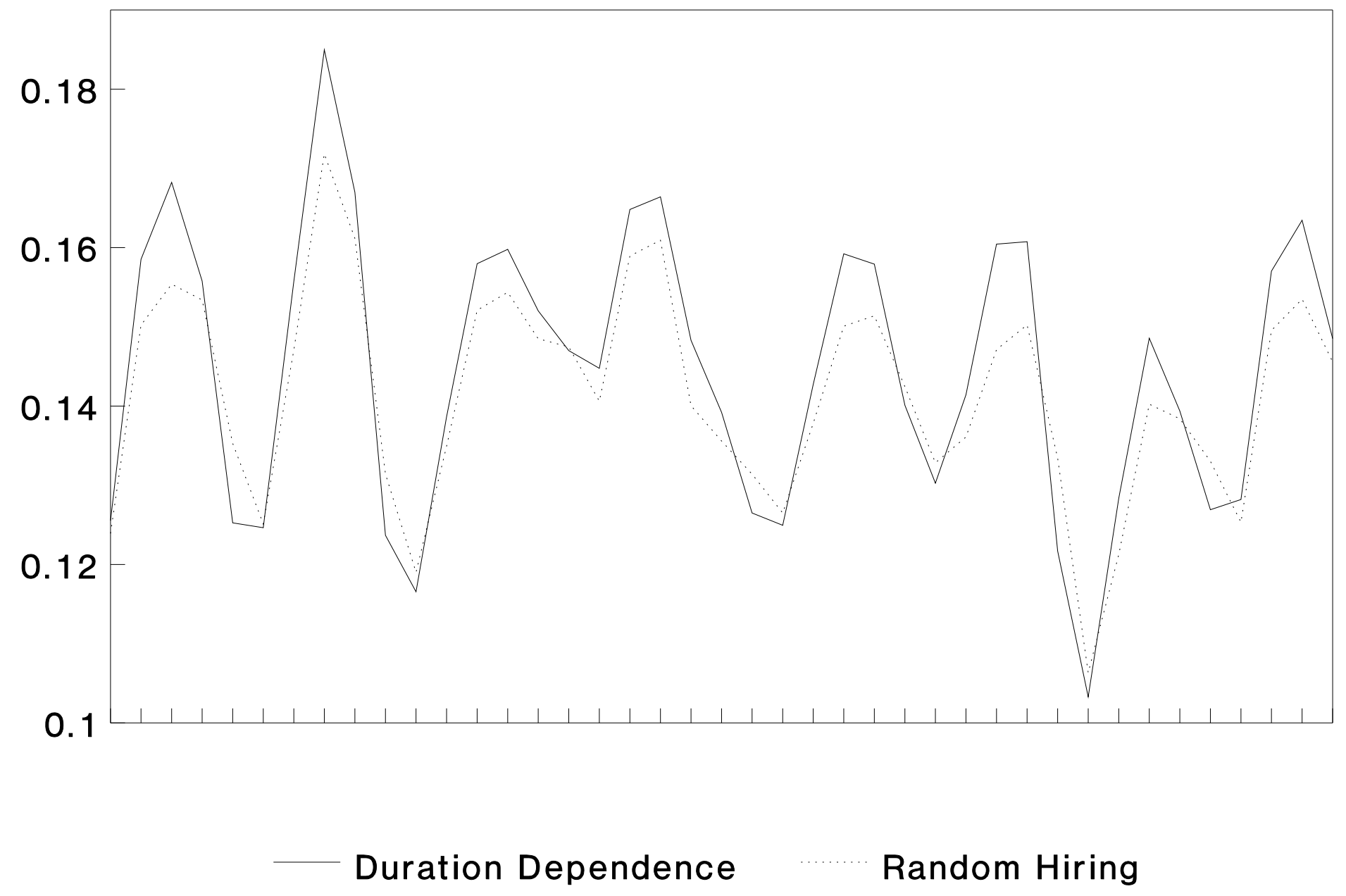

Figure 4: Unemployment Rate: Stochastic Business Cycle 


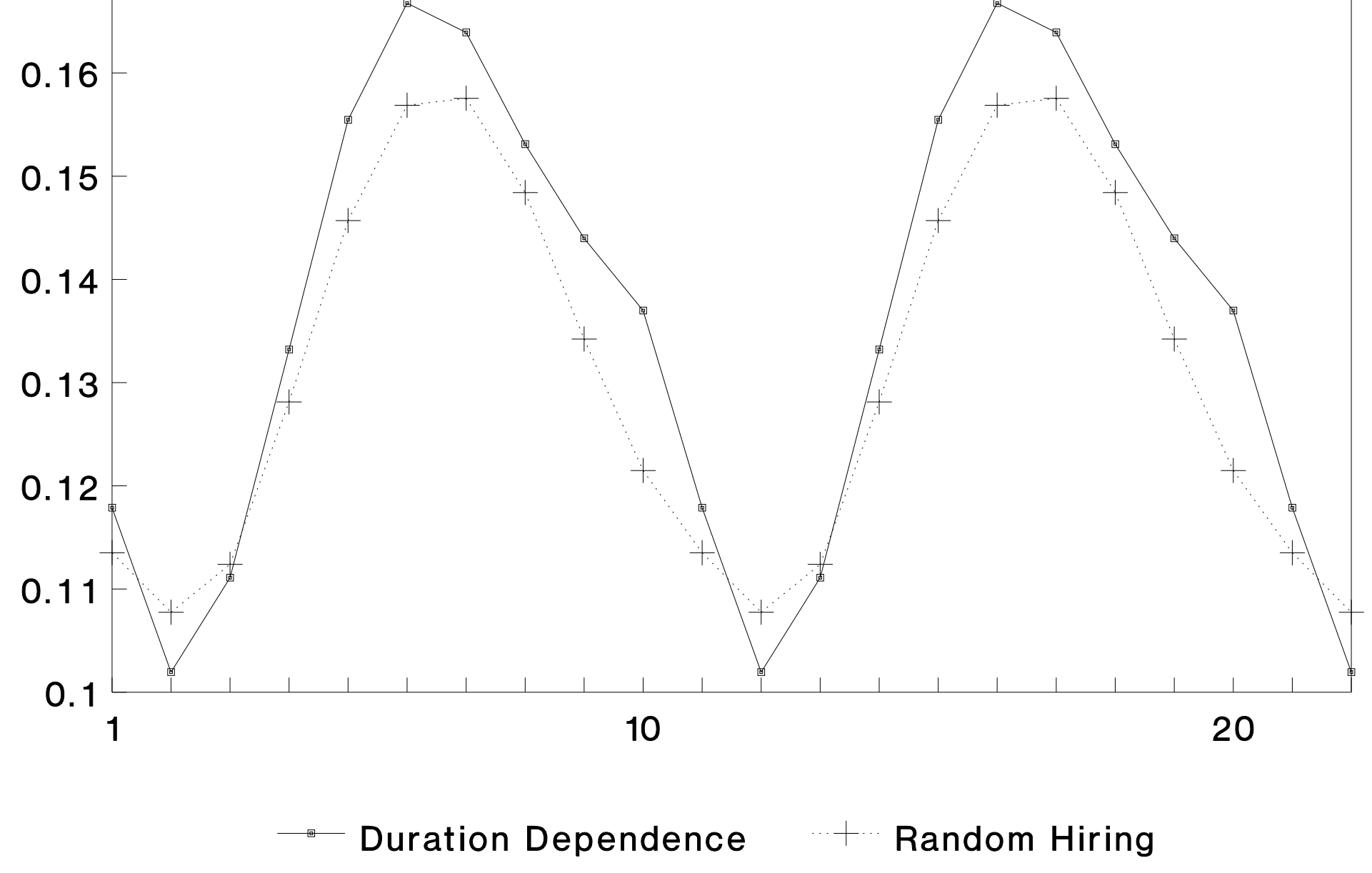

Figure 5: Unemployment Rate: Deterministic Cycle 